\title{
Exercise Training for Ameliorating Cardiovascular Risk Factors-focusing on Exercise Intensity and Amount
}

\author{
Satoru Kodama*, Shu Miao*, Nobuhiro Yamada ${ }^{* *}$ and Hirohito Sone ${ }^{*, * *}$ \\ *Doctoral Program in Sports Medicine, University of Tsukuba Graduate School of Comprehensive Human Sciences \\ 1-1-1 Tennodai, Tsukuba, Ibaraki 305-8575 Japan \\ hsone@md.tsukuba.ac.jp \\ ** Department of Internal Medicine, Sports Medicine, University of Tsukuba Institute of Clinical Medicine \\ 1-1-1 Tennodai, Tsukuba, Ibaraki 305-8575 Japan \\ [Received May 20, 2005 ; Accepted September 29, 2005]
}

\begin{abstract}
Exercise training suppresses cardiovascular risk factors by improving lipid/glucose metabolism, insulin resistance and hypertension. There is an optimum amount and intensity of exercise that will suppress each risk factor, although limited information is currently available on this area. For instance, the level of high-density lipoprotein cholesterol seems to be dependent more on the amount than on the intensity of exercise training; the situation with exercise-induced suppression of triglyceride levels is less clear. It is notable that the ability of mild intensity or a low amount of exercise to suppress or prevent cardiovascular risk factors is largely unknown. This knowledge is essential for prescribing safe and effective exercise training to elderly people. At the same time, very few studies on the associations between cardiovascular risk factors in response to exercise can be found. For example, improvement in lipid metabolism or insulin resistance seems to be related to the weight loss seen during exercise training but weight-independent effects could also be observed. The optimal intensity and amount of exercise training for each cardiovascular risk factor and the relationships between the effects of exercise training on each risk factor need to be clarified in the near future in order to establish guidelines that can be applied to the majority of elderly people at risk of cardiovascular diseases.
\end{abstract}

Keywords: cardiovascular risk factors, exercise intensity, exercise amount, elderly people

[International Journal of Sport and Health Science Vol.4, 325-338, 2006]

\section{Introduction}

It is known that obesity (especially increased abdominal fat), hypertension, hyperlipidemia, and insulin resistance are risk factors for cardiovascular disease (CVD) (DeFronzo, 1991; Carr, 2004; Rios, 1998; Goodpaster, 2005). Numerous reports have also shown that low cardiorespiratory fitness is a risk factor for CVD (Blair, 1991; Carnethon, 2003; Church, 2001; Lee, 1999; Farrell, 1998; Haddock, 1998; Lee, 2005). In general, exercise training ameliorates CVD risk factors through the effects shown in Table 1. Thus, exercise training would be expected to decrease the incidence of CVD in elderly people at high risk of CVD. The improvement in lipid metabolism and insulin resistance after exercise training, however, is inconclusive since there are potentially confounding variables to be considered when examining the effects of exercise, such as exercise intensity and amount, and exercise-related weight loss and reduction of body fat (Cox, 1994). In particular, there is no clear evidence that low level exercise training with potentially limited weight loss, which is feasible for the elderly, is effective in improving insulin resistance and hyperlipidemia.

Associations between the improvement in CVD risk factors are often considered when investigating the efficacy of exercise training in protecting against arteriosclerosis-related diseases. However, it remains unresolved as to whether there are cause and effect relationships between the improved risk factors. For example, it remains controversial whether a 
Table 1 CVD Risk Factor Improvement Effect by Exercise Training

1) Decrease in weight (or body fat including intra-abdominal body fat) ( Zelasko (1984), Poirier (2001))

2) Improvement of aerobic capacity (Vo2max, etc.) ( Samitz (1991))

3) Improvement of fat metabolism (HDL-C increase, TG decrease) ( Haskell WL (1984), Goldberg L (1987), Durstine (2001), Halbert (1999) )

4) Improvement of insulin resistance (fasting insulin concentration, HOMA-IR, and decrease of rate of glucose disappearance by insulin clamp technique) ( Henriksson(1985), Borghouts (2000), Hawley (2004))

5) Effect of hypotension (decrease of resting blood pressure) (Arroll (1992), Kelley (1999), Hagberg (2000), Fagard (1999), Stewart (2005))

reduction in insulin resistance is dependent on weight loss after a program of exercise training that results in reductions in both insulin resistance and obesity, or whether there are other factors that cause both improvements.

Our aim here is to review studies examining the effect of exercise training on CVD risk factors, notably those that focus on the effect of the intensity and amount of exercise and those that focus on the relationships between improved CVD risk factors. This will, hopefully, clarify the hitherto unsolved problems that need to be addressed before an effective exercise prescription for the majority of elderly people at high risk of CVD can be established.

\section{Effect of Exercise Training on Improving CVD Risk Factors}

It is generally held that exercise training improves CVD risk factors, as noted above. However, the improvements observed have not necessarily been consistent among studies and the amount and intensity of exercise are important confounding factors (Crouse, 1997).

\subsection{Effect on Serum Lipid}

In general, exercise training has a favorable effect on serum lipids such as high density lipoprotein cholesterol (HDL-C), which has a protective effect against arteriosclerosis by removing cholesterol from the peripheral tissues, and triglycerides (TG) which are contained in very low density lipoprotein (VLDL) (Haskell, 1984; Goldberg, 1987). Low density lipoprotein cholesterol (LDL-C) decreases in some situations but seem less amenable to change induced by exercise than HDL-C and TG (Cox, 1994).

\subsubsection{Effect on HDL-C}

A cross-sectional study has indicated that there is a correlation between daily physical activity and serum HDL-C or TG levels. Hata, et al. (2000) report that persons who walk more than 6000 steps daily on average have a higher HDL-C of $3 \mathrm{mg} / \mathrm{dl}$ and a lower TG of $10 \mathrm{mg} / \mathrm{dl}$ than those who walk less than 2000 steps. In addition, their meta-analysis also indicated that more intense exercise such as jogging or swimming resulted in a $10 \mathrm{mg} / \mathrm{dl}$ reduction in $\mathrm{TG}$ and a $5 \mathrm{mg} / \mathrm{dl}$ elevation in HDL-C together with an approximately $300 \mathrm{kcal}$ increase in energy expenditure per single bout of exercise. Interestingly, HDL-C was more significantly altered by exercise than any other lipoprotein measured (Szapary, 2003). Numerous studies have examined the effect of the intensity and amount of exercise on the change in HDL-C and some have suggested that there is a dose-dependent relationship between the amount of exercise and the increase in HDL-C, as shown in Table 2 (Wood, 1983; Kraus, 2002; Kokkinos, 1995).

In contrast, most studies suggest (Kokkinos, 1998 excepted) that there is not a strong association 
Table 2 Dose-dependent effect on HDL-C by regular exercise

\begin{tabular}{lll}
\hline presenter & subject & result \\
\hline Kokkinos (1995) & $\begin{array}{l}2906 \text { (mean age: } 43 \pm \\
4 y r s)\end{array}$ & $\begin{array}{l}\text { HDL-C increases by about 0.3mg/dl } \\
\text { as weekly jogging increases every } \\
\text { one mile. }\end{array}$ \\
Kraus (2002) & $\begin{array}{l}\text { 111 obese middle-aged } \\
\text { women (mean age: } \\
52 \pm 8 y r s)\end{array}$ & $\begin{array}{l}\text { Weekly 20-mile and 6-month jogging } \\
\text { increased 4.3mg/dl in HDL-C but } \\
\text { weekly 12-mile jogging did not } \\
\text { increase HDL-C. }\end{array}$ \\
& $\begin{array}{l}\text { When weekly running of more than } \\
\text { (mean age: } 45 y r s)\end{array}$ & $\begin{array}{l}\text { W miles for one year was continued, } \\
\text { the amount of weekly running } \\
\text { showed a significant correlation with } \\
\text { the change of HDL-C (r=0.48). }\end{array}$ \\
\hline
\end{tabular}

between exercise intensity and HDL-C increase (Spate-Douglas, 1999; Crouse, 1997; Kim, 2001). Resistance training is typically the form of high intensity training selected, although this form of exercise should be distinguished from endurance training. Joseph, et al. (1999) reported that resistance training resulted in a slight but significant increase of approximately $2 \mathrm{mg} / \mathrm{dl}$ in HDL-C (there was no significant change in TG) in middle-aged and elderly obese male subjects (aged 54-71yrs) after twelve weeks of resistance training. However, most studies showed no significant changes in HDL-C and TG concentrations after resistance training (Elliott, 2002; LeMura, 2000; Manning, 1991; Hurley, 1998). These findings suggest that exercise intensity is not strongly associated with the elevation of HDL-C. An increase in apolipoprotein (Apo) AI, which is the main constituent of HDL-C, is accompanied by an increase in HDL-C (Leon, 2002). Exercise intensity is not associated with the increase in HDL-C or ApoAI provided that energy expenditure is held constant (Crouse, 1995).

Exercise training-induced changes in HDL-C are affected by genetic polymorphisms of lipoprotein components such as ApoE, cholesteryl ester transfer protein (CETP), which alters HDL-C subtraction by transferring cholesterol from $\mathrm{HDL}_{2}$ to $\mathrm{HDL}_{3}$, and by genetic polymorphism of lipoprotein lipase (LPL), the enzyme that dissolves TG. Hagberg, et al. (1999), who determined whether the Apo E genotype affects the exercise training-induced increase in HDL-C, reported that men with the Apo E2 genotype had a larger increase in plasma HDL-C with exercise training than men with the Apo E3 and E4 genotype. Wilund, et al. (2002) determined whether variation in the CETP gene locus contributed to the variability in the responses of HDL-C to endurance exercise training. They reported that $\mathrm{HDL}_{2}$, which has the strongest effect in preventing arteriosclerosis among the HDL family, increased with endurance exercise training in CETP B1B1 but did not change in CETP B1B2 genotype individuals. In addition, Hagberg, et al. (1999), who used restriction fragment length polymorphism (RFLP) to examine the role of genotype in the response to exercise training, reported that LPL PvuII -/- individuals had larger increases in HDL-C than LPL PvuII +/- and +/+ individuals.

Familial factors also contribute significantly to the variability in the training response (Bouchard, 2001). However, Rice's results from the HERITAGE family study indicated that the percentage variance in the training responses attributable to familial factors ranged from $25 \%$ to $38 \%$ (Rice, 2002). Moreover, Leon, et al. (2002) reported that multivariate regression analysis including baseline variables and training responses only accounted for $15.5 \%$ of the variability in the HDL-C response to training. Molecular studies are currently underway to identify the markers and genes associated with training responses. 


\subsubsection{Effect on TG}

The reduction in blood TG levels after exercise is also well known (Haskell, 1984). As an acute response to exercise, the reduction in TG occurs about a day after one bout of exercise (Annuzzi, 1987; Sady, 1986; Cullinane, 1982) and persists for up to three days (Thompson, 1980). In these studies, the subjects took exercise for a long time (at least one-hour). Ferguson, et al. (1998) showed that the training subjects were required to consume at least 1000 -kcal during acute exercise to increase the activity of LPL. This may explain why high-volume exercise is required before acute reductions in TG are seen in adolescents and athletes. However, it is unclear whether an acute decline in TG would also occur after exercise in sedentary or elderly people who are incapable of high volume exercise. By contrast, exercise intensity was not found to affect the TG acute effect (Crouse, 1995).

It is also known that a chronic effect of exercise is the suppression of post-prandial hypertriglyceridemia. Merrill, et al. (1989) found that running more than 30 miles per week suppressed the post-prandial TG response by $34 \%$. However, it is controversial whether prolonged training reduces the fasting TG concentration. A meta-analysis of the influence of exercise training on fasting TG levels showed that energy expenditure of 1200 to $2200 \mathrm{kcal}$ per week dose-dependently reduced TG by 5 to 38 mg/dl (Durstine, 1991). However, Kelley's (2004) meta-analysis indicated that exercise training did not significantly reduce the fasting TG concentration (Kelley, 2004). Moreover, the decrease in TG was dispersed among individuals (Leon, 2001).

Clearly, both the acute and the chronic effects of exercise on TG levels need to be examined. Moreover, there are surprisingly few reports on the relationship between the amount and intensity of exercise and the change in TG levels. Further research is also necessary into the factors, including enzymatic activities, which affect the changes in HDL-C, TG and apoprotein.

\subsection{Effect on Carbohydrate Metabolism}

Insulin controls blood glucose levels (suppresses hyperglycemia) by promoting the uptake of post-prandial glucose by the liver, skeletal muscle, and fat tissue. Berson and Yalow (1970) defined insulin resistance as "a state (of a cell, tissue, system or body) in which greater than normal amounts of insulin are required to elicit a quantitatively normal response." Insulin resistance has been defined accurately by a decreased rate of glucose disappearance $(\mathrm{Rd})$ during a hyperinsulinemic-euglyc emic clamp (Defronzo, 1979). It is simply estimated by high levels of plasma insulin (either during fasting or during a glucose tolerance test) and homeostasis model assessment insulin resistance (HOMA-IR) [fasting plasma glucose $(\mathrm{mg} / \mathrm{dl}) \mathrm{x}$ fasting plasma insulin $(\mu \mathrm{U} / \mathrm{ml}) / 405]$ (Flier, 1993).

A high-intake of fat and obesity impair insulin-mediated glucose uptake (Vessby, 2000). Insulin resistance and/or the compensatory hyperinsulinemia will lead to dyslipidemia (i.e. high TG and low HDL-C) and high blood pressure, which plays an important role in causing type 2 diabetes or/and CHD (Reaven, 1988).

\subsubsection{Effect on Prevalence of Diabetes}

It is well known that daily physical activity protects against impaired carbohydrate metabolism. $\mathrm{Hu}$, et al. (1999) showed during an 8 year of follow up study that the intensity and amount of physical activity related to the incidence of type 2 diabetes. Three Randomized Control Trial (RCT) studies carried out in different countries indicated that the incidence of type 2 diabetes in high risk subjects with impaired glucose tolerance (IGT) was reduced by $41 \%$ to $58 \%$ through lifestyle intervention that included alterations to diet and physical activity (Pan, 1997; Tuomilehto, 2001; Knowler, 2002). Table 3 shows a summary of the three RCT study data.

\subsubsection{Effect on Carbohydrate Metabolism}

There are many reports that exercise training improves carbohydrate metabolism in type 2 diabetes, including improving glycemic control (i.e. lowering $\mathrm{HbA}_{1} \mathrm{c}$ ). Boule, et al. (2001) reported that the weighted mean post-intervention $\mathrm{HbA}_{1} \mathrm{c}$ was lower by $-0.66 \%$ in the exercise groups compared with the control groups, although no significantly greater change in body mass was found by their meta-analysis when exercise groups were compared with control groups.

Although there is little evidence on the impact of the amount and intensity of aerobic exercise on glycemic control, the decrease in $\mathrm{HbA}_{1} \mathrm{c}$ may have a stronger association with exercise intensity than with exercise amount. Kelley's (2001) meta-analysis 
Table 3 Primary Preventive Clinical Test of main type 2 diabetes

\begin{tabular}{|c|c|c|c|}
\hline Study & $\begin{array}{c}\text { Da Qing IGT and } \\
\text { Diabetes Study, } \\
\text { China } \\
\text { ( Pan (1997) })\end{array}$ & $\begin{array}{c}\text { Finnish Diabetes } \\
\text { Prevention Study } \\
\text { ( Tuomilehto (2001)) }\end{array}$ & $\begin{array}{c}\text { Diabetes Prevention } \\
\text { Program, USA } \\
\text { ( Knowler }(2002))\end{array}$ \\
\hline $\begin{array}{l}\text { Number of } \\
\text { subjects }\end{array}$ & 520 & 522 & 3234 \\
\hline $\begin{array}{l}\text { Rate of } \\
\text { women (\%) }\end{array}$ & 47 & 67 & 68 \\
\hline Mean age & $45 \pm 9$ & $55 \pm 7$ & $51 \pm 11$ \\
\hline $\begin{array}{l}\text { Mean body } \\
\text { mass index }\end{array}$ & $25.8 \pm 3.8$ & $31.2 \pm 4.6$ & $34.0 \pm 6.7$ \\
\hline $\begin{array}{l}\text { Period of } \\
\text { intervention } \\
\text { (year) }\end{array}$ & 6 & 3.2 & 2.8 \\
\hline Group & $\begin{array}{l}\text { Control group } \\
\text { Eating group } \\
\text { (25-30kcal/day, } \\
\text { limiting fat intake } \\
\text { (25-30\% of total } \\
\text { calories) } \\
\text { Exercise group } \\
\text { (exercise for } \\
\text { 1-2hrs/day) } \\
\text { Eating and } \\
\text { exercising group }\end{array}$ & $\begin{array}{l}\text { Control group } \\
\text { Lifestyle } \\
\text { intervention group } \\
\text { (limiting fat intake } \\
\text { (MAX 30\% of total } \\
\text { calories), targeting } \\
5 \% \text { decrease in the } \\
\text { original weight by } \\
\text { exercise of more than } \\
\text { 30min/day) }\end{array}$ & $\begin{array}{l}\text { Control group } \\
\text { Medication group } \\
\text { Lifestyle } \\
\text { intervention group } \\
\text { (to decrease by } 5-7 \% \\
\text { the original weight } \\
\text { by order-made } \\
\text { exercise and dietary) }\end{array}$ \\
\hline $\begin{array}{l}\text { Result } \\
\text { (decrease } \\
\text { rate of } \\
\text { development } \\
\text { of type } 2 \\
\text { diabetes) }\end{array}$ & $\begin{array}{l}\text { Eating group:31\% } \\
\text { Exercising group: } \\
46 \% \\
\text { Eating+Exercising } \\
\text { group: } 42 \%\end{array}$ & $\begin{array}{l}\text { Lifestyle } \\
\text { intervention group: } \\
58 \%\end{array}$ & $\begin{array}{l}\text { Medication group: } \\
31 \% \\
\text { Lifestyle } \\
\text { intervention } \\
\text { group: } 58 \%\end{array}$ \\
\hline
\end{tabular}

Body Mass Index is calculated by (weight (kg)/height2 (m2)).

Criteria of obesity is more than $25(\mathrm{~kg} / \mathrm{m} 2)$.

showed that it is uncertain whether there is a dose-response effect of exercise on improved glucose control in Type 2 diabetes. Boule's (2003) meta-analysis of the improvements in glycemic control after aerobic training indicated that exercise intensity predicted the post-intervention improvement in $\mathrm{HbA}_{1} \mathrm{c}(r=-0.91, p=0.002)$ to a larger extent than did exercise duration.

Lately, there have been an increasing number of studies on the usefulness of resistance training in 
improving glycemic control and insulin resistance in diabetic patients. Resistance training alone (Dunstan, 2005; Castaneda, 2002; Dunstan, 2002; Ishii, 1998; Honkola, 1997) and combined with aerobic training (Cuff, 2003; Maiorana, 2002) was found to be effective in improving glycemic control and insulin resistance. Resistance training is also known as high intensity training although its exercise form is different from endurance training. The studies of resistance training may suggest that exercise intensity is strongly associated with the improvement of carbohydrate metabolism in diabetic patients. However, to the best of our knowledge, the amount and intensity of resistance training required to elicit an improvement in carbohydrate metabolism has never been studied. Further research on the design of a safe resistance-training program for the purposes of preventing and caring for type 2 diabetes is necessary.

\subsubsection{Effect on Insulin Resistance (Sensitivity)}

It is well known that physical activity and exercise training reduce insulin resistance (Ross, 2004; Dumortier, 2003; Boudou, 2003). In fact, some cross-sectional studies have shown that a low level of physical activity is associated with insulin resistance (Saito, 1992; Harding, 2001). However, there have been few exercise intervention studies of the intensity and amount of exercise required for improving insulin resistance and the findings of these studies were disparate. Borghouts, et al. (1999) reported that improvements in insulin sensitivity in healthy young adults after four weeks' endurance training were more pronounced with high intensity training. Resistance training also has been shown to be effective in the improvement of insulin resistance in elderly people and menopausal women (Reynolds, 2004; Ryan, 2000; Ryan, 1996). These results would indicate that exercise intensity is related to an improvement in insulin resistance. By contrast, Houmard's findings in obese individuals suggested that total exercise duration per week and not exercise intensity should considered when designing training programs with the intent of improving insulin action (Houmard, 2004). Mayer, et al. (1998) studied exercise training by 1467 African American females and found that the amount and intensity of exercise were associated with higher insulin sensitivity. Thus, both the amount and the intensity of exercise may be associated with the improvement in insulin resistance, although the relative contribution of each factor is unknown at present.

Another factor relating to the improvement in insulin resistance after exercise training is age. Short (2003), who examined the impact of exercise training at 70-80\% maximum heart rate (HR max) on improving insulin resistance in subjects of 21-87yrs, showed that increase in insulin sensitivity is adversely correlated with age $(r=-0.41)$. It is possible that, for a given level of exercise intensity, the effect of exercise training on the improvement in insulin sensitivity declines with age.

\subsection{Effect on Resting Blood Pressure}

A large-scale epidemiological survey indicated that moderately vigorous physical activity led to a reduced risk of hypertension (Paffenbarger, 1997; Hernelahti, 2004) while the volume of physical activity (including light physical activity) was not associated with hypertension (Hernelahti, 2004).

The findings of exercise intervention studies have been inconsistent with regard to the effect of endurance training intensity in lowering blood pressure. Cooper, et al. (2000) reported that the magnitude of the hypotensive effect of moderate intensity exercise was not as great as that found in studies of higher intensity exercise among hypertensives (systolic blood pressure (SBP): $1.9 \mathrm{mmHg}$, diastolic blood pressure (DBP): -2.2 $\mathrm{mmHg}$ ). On the contrary, Motoyama, et al. (1997) reported that SBP $(-15+/-8 \mathrm{~mm} \mathrm{Hg})$ and DBP $(-9$ $+/-9 \mathrm{~mm} \mathrm{Hg}$ ) decreased significantly after 3 months of lactate threshold (LT) level intensity exercise training in elderly hypertensive subjects. Braith, et al. (1994) reported that SBP decreased similarly in both moderate intensity ( $70 \%$ heart rate reserve (HRR)) and high intensity ( $85 \%$ HRR) training groups of elderly people after 6 months. Halbert' s meta-analysis of 29 studies of exercise training by the elderly reported that exercise training of more than $70 \%$ maximum oxygen consumption $\left(\mathrm{VO}_{2}\right.$ max) intensity did not have any additional impact on reducing BP (Halbert, 1997). These reports may suggest that high intensity training is not necessarily required in order to lower resting BP in elderly people, although it is unclear if low intensity training (e.g. less than LT intensity) also has a hypotensive effect.

In fact, resistance training as a typical form of high intensity training is not recommended for 
the treatment of hypertension (Pescatello, 2004). The acute and chronic influences of resistance training on blood pressure have been examined in normotensive individuals. The results suggest that resistance training is not associated with chronic elevations in blood pressure, but could become part of a non-pharmacological intervention strategy to prevent and combat high blood pressure (Schwartz, 1995). In general, blood pressure elevates during resistance exercise. In normotensive individuals, the mean blood pressures declined during the hour after resistance training (Fisher, 2001) and the average values for 24-h ambulatory blood pressure pre- and post-training did not differ (Gilders, 1991). Cornelissen, et al. (2005) determined the influence of resistance training in healthy normotensive adults by meta-analysis and found that the weighted net change (SBP/DBP) in blood pressure averaged $-3.2 /-3.5$ $\mathrm{mmHg}$. Kelley (2000) also reported that resistance training resulted in decreases of approximately 3 $\mathrm{mmHg}$ in both resting SBP and resting DBP. It is concluded that moderate intensity resistance training is not harmful for normotensive subjects. However, additional studies are needed in hypertensive subjects before resistance exercise can be considered as a safe and effective regimen of therapy.

The dose-response relationship between exercise training and the change in blood pressure has been examined by some studies. Ishikawa, et al. (2003) reported that the magnitude of reductions in SBP was greater in the 61 to $90 \mathrm{~min} / \mathrm{wk}$ group than in the 30 to $60 \mathrm{~min} / \mathrm{wk}$ group, but there were no greater reductions in SBP with further increases in exercise volume. Halbert, et al. (1997) reported that increasing exercise frequency to more than three sessions per week did not have any additional impact on reducing blood pressure. Thus, the amount of exercise may have comparatively little effect on the reduction of blood pressure.

\section{Correlation between Risk Factor Improvements}

As mentioned above, little is known about the cause and effect relationships between exercise and the improvements in individual CVD risk factors. Previous reports have only examined the correlations between exercise training-induced improvements in risk factors (Miyatake, 2002; Mourier, 1997; Ardern, 2004; Despres, 1991; Leon, 2000; Poirier,
2001; Miyatake, 2003). Most of these reports focussed on the correlation between the improvement in fat metabolism and insulin resistance and the improvement in body composition and aerobic capacity after training. Moreover, few studies have examined whether the concurrent improvements in insulin resistance and fat metabolism observed with exercise training are independent of exercise-induced changes in body weight and aerobic capacity (Lamarche, et al., 1992).

\subsection{Correlation between Reduction of Obesity and Improvement of Fat Metabolism/Insulin Resistance}

Some studies have reported that an improvement in lipid metabolism and insulin resistance after exercise training relates to a reduction in body fat (or visceral fat). Miyatake, et al. (2002) has shown using stepwise multiple regression analysis that the change in visceral fat area was the major determinant for the change in HOMA-IR $(r=0.51)$ in obese middle-aged males. Mourier, et al. (1997) reported that the change in visceral abdominal fat was associated with the improvement in insulin sensitivity $(r=0.84)$ in type 2 diabetic patients after aerobic training at $75 \% \mathrm{VO}_{2}$ peak. Ardern, et al. (2004) found in the HERITAGE family study (17-65 yrs) that greater fat loss was associated with increases in HDL-C and decreases in TG after exercise training.

It is controversial whether exercise training itself improves blood lipid levels or insulin resistance regardless of weight loss. Some studies have shown that aerobic training results in a decrease in TG (Weintraub, 1989) and a modest increase in HDL-C (Thompson, 1988) without weight loss in healthy subjects. Duncan, et al. (2003) reported that in obese,

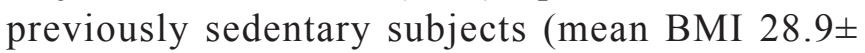
$4.6 \mathrm{~kg} / \mathrm{m}^{2}$ ), walking exercise positively affected markers of glucose and fat metabolism even in the absence of weight loss. Most reports, however, suggest that weight loss by exercise training and/or caloric restriction should be encouraged to improve the CHD risk factor profile of obese men (Ross, 2000; Katzel, 1997). Wood, et al. (1988) reported that the changes in plasma lipids and lipoproteins induced by decreasing energy intake or by increasing exercise were not significantly different in obese persons. These reports suggest that improvements in lipid metabolism and insulin resistance are strongly 


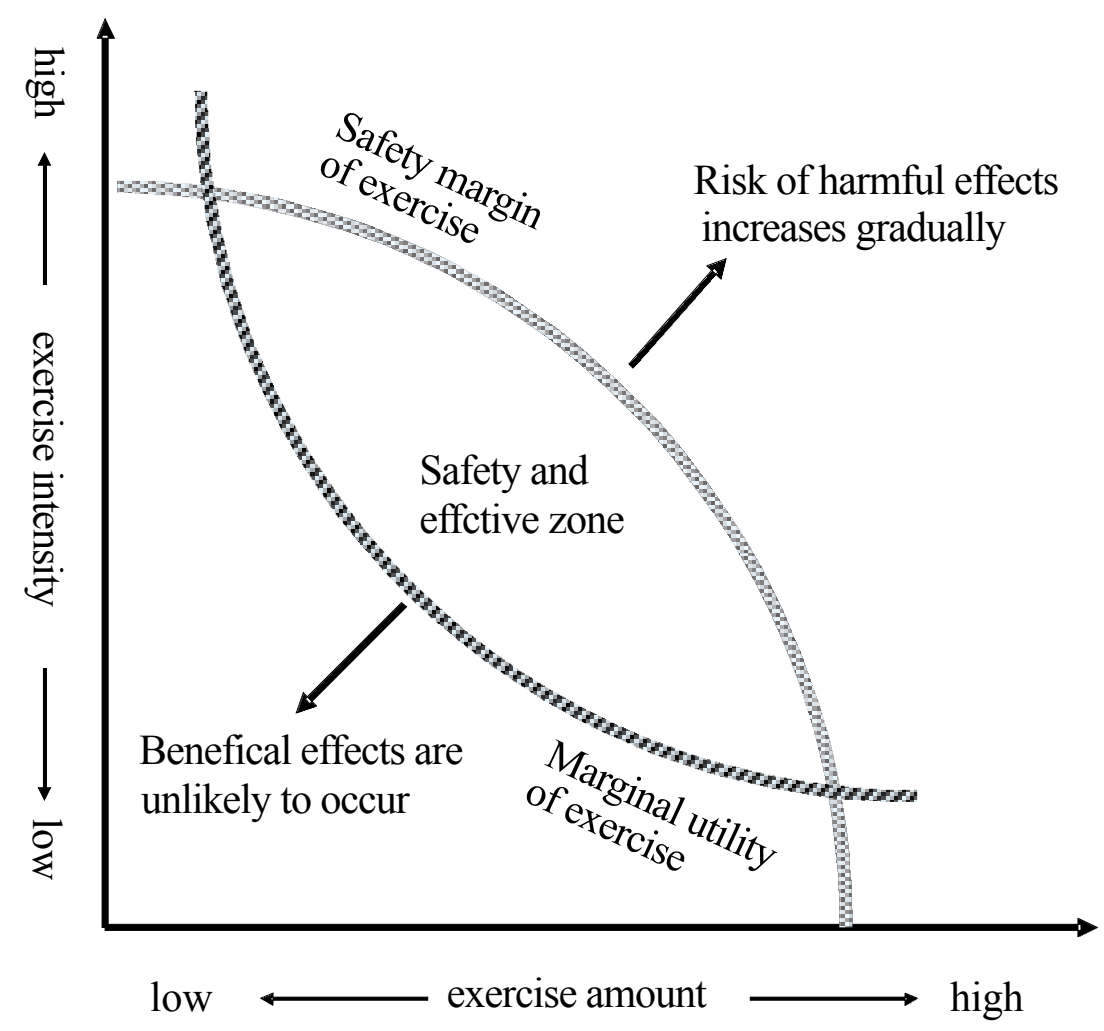

Figure 1 Hypothetical safe therapeutic margin in exercise prescription for improving cardiovascular risk factors

associated with the loss of body weight/fat. Exercise training itself may be effective in ameliorating CHD risk factors; however it is more important for obese persons to reduce obesity to improve fat metabolism and insulin resistance.

\subsection{Relationships between Improvement of Aerobic Capacity and Improvement of Fat Metabolism and Insulin Resistance}

The relationship between the improvement of aerobic capacity and the improvement of fat metabolism and insulin resistance has been examined in many studies. Gan (2003) excepted, there is a clear dissociation between the improvement in cardiorespiratory fitness and the improvement in the metabolic risk profile after endurance training programs (Despres, 1991; Leon, 2000; Poirier, 2001). Regular exercise training is likely to generate beneficial metabolic effects regardless of the improvement of aerobic function.

\subsection{The Relationship between the Reduction of Obesity and Blood Pressure}

In a meta-analysis, Neter, et al. (2003) estimated the effect of weight reduction on blood pressure and indicated that SBP and DBP reductions were $1.05 \mathrm{mmHg}$ and $-0.92 \mathrm{mmHg}$, respectively, when expressed per kilogram of weight loss. Miyatake, et al. (2003), who examined the link between a reduction in blood pressure (BP) and daily exercise, reported that there was a positive correlation $(r=0.47)$ between the reduction in DBP and the decrease in visceral fat area measured with computed tomography (CT) scanning. Blumenthal, et al. (2000), however, reported that both weight loss and aerobic exercise were associated with a reduction in blood pressure. From these reports, it can be concluded that exercise training alone may have an antihypertensive effect, although weight loss is important for reducing blood pressure. Little is known about how much exercise training and weight loss, respectively, contribute to lowering blood pressure.

\subsection{Relationships between the Improvement in Aerobic Capacity and the Reduction in Blood Pressure}

Some studies have shown that an increase in aerobic fitness does not coincide with a reduction 


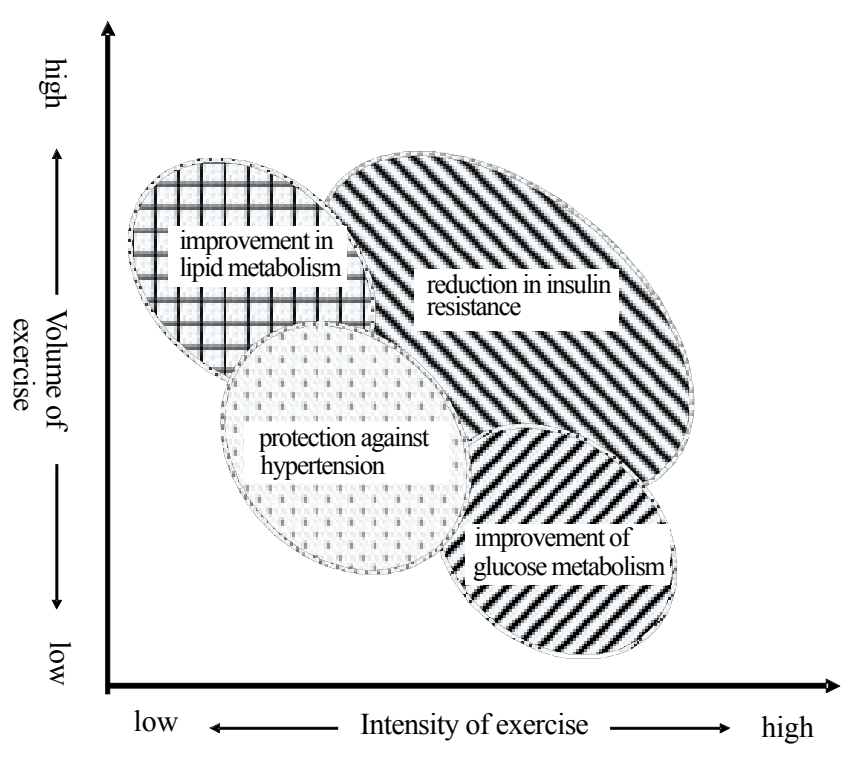

Figure 2 An illustration of the intensity and amount of exercise which is optimal for the improvement of each CVD risk factor (it does not show quantitative relationships between each areas.)

in blood pressure (Grant, 1992; Sedgwick, 1989). There are fewer reports on the relationship between the changes in aerobic capacity and blood pressure than on the relationship between the improvements in aerobic capacity and fat metabolism and/or insulin resistance. Future investigations are necessary, therefore, on the relationship between exercise and the reduction in blood pressure.

\section{Exercise Training for Elderly People}

\subsection{Exercise Prescription for Elderly People}

Many studies have indicated that exercise training for elderly people is also effective in reducing insulin resistance (Seal, 1984; Kirman, 1993; Dengel, 1998; Reynolds $4^{\text {th }}$, 2002; Evans, 2005), improving lipid metabolism (Seal, 1984; Schuit, 1998; Fahlman, 2002; Park, 2003), and lowering blood pressure (Jessup, 1998, 1999; Kelley, 2001; Ohkubo, 2001). Thus, daily exercise training is expected to lower the incidence of CVD.

More than half of older people complain of barriers to physical activity. The common barriers are health issues such as heart and orthopedic problems (Lim, 2005). Consequently, little of the current research on the impact of physical activity on strength and function in elderly people has included elderly participants who have more barriers to physical activity (Taunton, 1997). Given that the elderly person is more fragile and has more problems than the middle-aged participant, the exercise intensity of the program is usually lower. With elderly people who are frail and/or have physical-medical limitations, exercise intensity may be decreased (Pollock, 1994). The American College of Sports Medicine (2004) has stated key practices for promoting physical activity, especially in older adults with chronic disease or low fitness and in those with low levels of physical activity. The key practices include the management of risk by commencing physical activity at low intensity and gradually increasing it. The amount of exercise may also be low because the elderly are affected by the drawbacks of physical overloading and the safety margin of an exercise dose tends to decline with aging. However, there are few studies on the efficacy of a low amount or a low intensity of exercise training in elderly people.

\subsection{Low Intensity Training for Elderly People}

Low intensity (e.g. $50 \% \mathrm{VO}_{2} \max$ ) exercise training has also been shown to be effective in enhancing aerobic capacity in some studies. De Vito, et al. (1997) reported that exercise training at 50\% HRR did not result in a significant improvement in $\mathrm{VO}_{2}$ max. However, most studies reported that aerobic capacity was improved even by low intensity training. Poster, et al. (1992) reported that $\mathrm{VO}_{2}$ max was increased by $8.5 \%$ by low to moderate intensity (70\%HR max) exercise training. It also has been found that low (30-40\% HRR) and moderate intensity $(60-70 \% \mathrm{HRR})$ training produced similar improvements in $\mathrm{VO}_{2} \max$ (Stevenson, 1990; Foster, 1989). However, there are only a few reports that low intensity training by elderly people produces improvements in fat metabolism, insulin resistance, and resting blood pressure (Sunami, 1999; Stewart, 2005). Moreover, the effect of low intensity training (i.e. training which the subjects may feel is 'light') with perceived exertion as an indicator of somatic stress (Borg, 1970) has never been studied. The amount of exercise may need to be low for elderly people who are incapable of high volume training, as noted above. When designing a training program for the elderly who have barriers to high volume exercise, a confounding problem is quantification of the dose-response relationships between the volume 
of exercise and the improvements in risk factors. It is currently unclear how many bouts of exercise are required to ameliorate individual risk factors in the elderly.

There are a few reports on the correlation between the favorable effects of exercise on cardiovascular risk factors in elderly people (i.e. the correlation between weight loss and the improvement in aerobic capacity and fat metabolism and insulin resistance in exercise training). Katzel, et al. (1995) indicated that there was a significant correlation between the loss of body fat and the improvement in blood TG and HDL-C levels and insulin resistance. On the contrary, there was no significant association between the change in $\mathrm{VO}_{2}$ max and the reduction in insulin response during an oral glucose tolerance test (OGTT) after exercise training (Pratray, 2000). However, there are few reports on the relationships between these changes after low intensity exercise training which feasible for elderly people (Stewart, 2005). It is necessary to establish an exercise prescription which is not only effective in ameliorating CVD risk factors such as dyslipidemia, insulin resistance, hypertension but which is also feasible for the majority of elderly people. Future research is needed to determine the efficacy of low-intensity and low-amount exercise training and to determine the relationships between exercise and the improvements in CVD risk factors.

\section{Conclusion}

Generally, exercise training is effective in improving cardiovascular risk factors such as hyperlipidemia (e.g. low levels of HDL-C and high levels of TG), insulin resistance (abnormalities in carbohydrate metabolism), and hypertension. The improvements are affected by the amount and intensity of training (Figure 2) and by the degree of reduction in obesity and the increase in aerobic capacity. Other as yet unknown factors may also exist.

The increase in HDL-C, which is protective from arteriosclerosis, is affected more by the amount rather than the intensity of training. Resistance training, which is a typical form of high intensity training but which differs from endurance training, is not effective in elevating HDL-C. The improvement in insulin resistance is associated with both the intensity and the amount of exercise. On the contrary, an improvement in glycemic control in diabetes is associated more with exercise intensity than exercise amount. Moderate to high intensity exercise training (e.g. more than LT intensity) has been reported to be effective in the protection from hypertension. Nevertheless, it is unclear whether low intensity training (e.g. less than LT intensity) also has a hypotensive effect. An excessive amount of training is not necessarily required to reduce blood pressure. Most of the previous studies examining the relationships between exercise and the improvements in cardiovascular risk factors after exercise training suggest that the improvement in fat metabolism and insulin resistance is associated more with weight and/or fat loss rather than with enhanced aerobic capacity. A reduction in blood pressure through exercise training is also affected more by a reduction in body weight and/or fat than by improved aerobic capacity, although exercise training itself can also have a hypotensive effect regardless of weight loss.

Since the physical condition of the elderly population is variable, there are limited studies on exercise training in elderly subjects including those who are not physically active. In particular, there have been few reports on the improvements in CHD risk factors after low-intensity/amount exercise training which is feasible for the majority of elderly people. In order to establish an exercise prescription that is optimal for the elderly, it is necessary to investigate the effect of exercise designed for the elderly on the risk factors for CHD.

\section{References}

American College of Sports Medicine. (2004). Physical activity programs and behavior counseling in older adult populations. Med Sci Sports Exerc. 36(11):1997-2003.

Annuzzi G. (1987). Jansson E, Kaijser L, Holmquist L, Carlson LA. Increased removal rate of exogenous triglycerides after prolonged exercise in man: time course and effect of exercise duration. Metabolism. 36(5):438-43.

Ardern CI. (2004). Race and sex similarities in exercise-induced changes in blood lipids and fatness. Med Sci Sports Exerc. 36(9):1610-5

Arroll B. (1992). Does physical activity lower blood pressure: a critical review of the clinical trials. J Clin Epidemiol. 45(5):439-47.

Blair SN. (1989). Physical fitness and all-cause mortality. A prospective study of healthy men and women. JAMA. 262(17):2395-401.

Blumenthal JA (2000). Exercise and weight loss reduce blood pressure in men and women with mild hypertension: effects on cardiovascular, metabolic, and hemodynamic functioning.Arch Intern Med. 160(13):1947-58

Borg G. (1970). Perceived exertion as an indicator of somatic 
stress. Scand J Rehabil Med. 2(2):92-8.

Borghouts LB. (1999). Effect of training intensity on insulin sensitivity as evaluated by insulin tolerance test. Eur J Appl Physiol Occup Physiol.80(5):461-6.

Bouchard C. (2001). Individual differences in response to regular physical activity. Med Sci Sports Exerc. 33(6 Suppl):S446-51; discussion S452-3.

Boudou P. (2003). Absence of exercise-induced variations in adiponectin levels despite decreased abdominal adiposity and improved insulin sensitivity in type 2 diabetic men. Eur J Endocrinol. 149(5):421-4.

Boule NG. (2001). Effects of exercise on glycemic control and body mass in type 2 diabetes mellitus: a meta-analysis of controlled clinical trials. JAMA. 286(10):1218-27.

Boule NG. (2003). Meta-analysis of the effect of structured exercise training on cardiorespiratory fitness in Type 2 diabetes mellitus. Diabetologia. 46(8):1071-81.

Braith RW. (1994). Moderate- and high-intensity exercise lowers blood pressure in normotensive subjects 60 to 79 years of age. Am J Cardiol. 73(15):1124-8.

Butler RN. (1998). Physical fitness: exercise prescription for older adults. 3. Geriatrics. 1998 53(11):45-6, 49-50, 52-4.

Carnethon MR. (2003). Cardiorespiratory fitness in young adulthood and the development of cardiovascular disease risk factors. JAMA.290(23):3092-100

Carr MC. (2004). Abdominal obesity and dyslipidemia in the metabolic syndrome: importance of type 2 diabetes and familial combined hyperlipidemia in coronary artery disease risk. J Clin Endocrinol Metab. 89(6):2601-7. Review.

Castaneda C (2002). A randomized controlled trial of resistance exercise training to improve glycemic control in older adults with type 2 diabetes. Diabetes Care. 25(12):2335-41.

Church TS (2001). Usefulness of cardiorespiratory fitness as a predictor of all-cause and cardiovascular disease mortality in men with systemic hypertension. Am J Cardiol. 88(6):651-6.

Cooper AR. (2000). What is the magnitude of blood pressure response to a programme of moderate intensity exercise? Randomised controlled trial among sedentary adults with unmedicated hypertension. Br J Gen Pract. 50(461):958-62.

Cornelissen VA. (2005). Effect of resistance training on resting blood pressure: a meta-analysis of randomized controlled trials. J Hypertens. 23(2):251-9.

Cox CM. (1994). Interaction between diet, exercise and lipids or lipoproteins. Curr Opin Lipidol.5(1):29-35.

Crouse SF. (1995). Changes in serum lipids and apolipoproteins after exercise in men with high cholesterol: influence of intensity. J Appl Physiol. 79(1):279-86.

Cuff DJ. (2003). Effective exercise modality to reduce insulin resistance in women with type 2 diabetes. Diabetes Care. 26(11):2977-82.

Cullinane E. (1982). Acute decrease in serum triglycerides with exercise: is there a threshold for an exercise effect? Metabolism. 31(8):844-7.

De Vito G. (1997). Low intensity physical training in older subjects. J Sports Med Phys Fitness. 37(1):72-7.

DeFronzo RA (1979). Glucose clamp technique: a method for quantifying insulin secretion and resistance. Am J Physiol. 237(3):E214-23.

DeFronzo RA. (1991). Insulin resistance. A multifaceted syndrome responsible for NIDDM, obesity, hypertension, dyslipidemia, and atherosclerotic cardiovascular disease. Diabetes Care. 14(3):173-94. Review
Despres JP. (1991). Loss of abdominal fat and metabolic response to exercise training in obese women. Am J Physiol. 261(2 Pt 1):E159-67.

Dumortier M. (2003). Low intensity endurance exercise targeted for lipid oxidation improves body composition and insulin sensitivity in patients with the metabolic syndrome. Diabetes Metab. 29(5):509-18.

Duncan GE. (2003). Exercise training, without weight loss, increases insulin sensitivity and postheparin plasma lipase activity in previously sedentary adults. Diabetes Care. 26(3):557-62.

Dunstan DW. (2002). High-intensity resistance training improves glycemic control in older patients with type 2 diabetes. Diabetes Care. 25(10):1729-36.

Durstine JL. (2001). Blood lipid and lipoprotein adaptations to exercise: a quantitative analysis. Sports Med. 31(15):1033-62. Review.

Elliott KJ. (2002). Effects of resistance training and detraining on muscle strength and blood lipid profiles in postmenopausal women. Br J Sports Med. 36(5):340-4.

Fagard RH. (1999). Physical activity in the prevention and treatment of hypertension in the obese. Med Sci Sports Exerc. 31(11 Suppl):S624-30.

Farrell SW. (1998). Influences of cardiorespiratory fitness levels and other predictors on cardiovascular disease mortality in men. Med Sci Sports Exerc. 1998 Jun;30(6):899-905.

Ferguson MA. (1998). Effects of four different single exercise sessions on lipids, lipoproteins, and lipoprotein lipase. J Appl Physiol. 85(3):1169-74.

Fisher MM. (2001). The effect of resistance exercise on recovery blood pressure in normotensive and borderline hypertensive women. J Strength Cond Res. 15(2):210-6.

Flier JS. (1993). An overview of insulin resistance. In Insulin Resistance. Moller DE, Ed. Chichester, U.K., Wiley, p. 1-8

Foster VL. (1989). Endurance training for elderly women: moderate vs low intensity. J Gerontol. 44(6):M184-8.

Gan SK. (2003). Changes in aerobic capacity and visceral fat but not myocyte lipid levels predict increased insulin action after exercise in overweight and obese men. Diabetes Care. 26(6):1706-13.

Gilders RM. (1991). The effect of resistance training on blood pressure in normotensive women. Clin Physiol. 11(4):307-14.

Goldberg L. (1987). Elliot DL. The effect of exercise on lipid metabolism in men and women. Sports Med. 1987 Sep-Oct;4(5):307-21.

Goodpaster BH. (2005). Obesity, regional body fat distribution, and the metabolic syndrome in older men and women. Arch Intern Med. 165(7):777-83.

Grant S. (1992). The effects of a university fitness programme on health-related variables in previously sedentary males. Br J Sports Med. 1992 26(1):39-44.

Haddock BL. (1998). Cardiorespiratory fitness and cardiovascular disease risk factors in postmenopausal women. Med Sci Sports Exerc. 30(6):893-8.

Hagberg JM. (1999). Exercise training-induced blood pressure and plasma lipid improvements in hypertensives may be genotype dependent. Hypertension.34(1):18-23.

Hagberg JM. (1999). Apolipoprotein E genotype and exercise training-induced increases in plasma high-density lipoprotein (HDL)- and HDL2-cholesterol levels in overweight men. Metabolism. 48(8):943-5.

Hagberg JM. (2000). The role of exercise training in the 
treatment of hypertension: an update. Sports Med. 30(3):193-206. Review.

Halbert JA. (1997). The effectiveness of exercise training in lowering blood pressure: a meta-analysis of randomised controlled trials of 4 weeks or longer. J Hum Hypertens. 11(10):641-9.

Halbert JA. (1999). Exercise training and blood lipids in hyperlipidemic and normolipidemic adults: a meta-analysis of randomized, controlled trials. Eur J Clin Nutr. 53(7):514-22.

Harding AH. (2001). Is the association between dietary fat intake and insulin resistance modified by physical activity? Metabolism. 50(10):1186-92.

Haskell WL. (1984). The influence of exercise on the concentrations of triglyceride and cholesterol in human plasma. Exerc Sport Sci Rev. 12:205-44.

Hata Y. (2000). Life-style and serum lipids and lipoproteins. J Atheroscler Thromb. 7(4):177-97.

Hawley JA. (2004). Exercise as a therapeutic intervention for the prevention and treatment of insulin resistance. Diabetes Metab Res Rev. 20(5):383-93.

Henriksson J. (1995). Influence of exercise on insulin sensitivity. J Cardiovasc Risk. 2(4):303-9.

Hernelahti M. (2004). Stability and change of volume and intensity of physical activity as predictors of hypertension. Scand J Public Health. 32(4):303-9.

Honkola A. (1997). Resistance training improves the metabolic profile in individuals with type 2 diabetes. Acta Diabetol. 34(4):245-8.

Houmard JA. (2004). Effect of the volume and intensity of exercise training on insulin sensitivity. J Appl Physiol.96(1):101-6.

Hu FB. (1999). Walking compared with vigorous physical activity and risk of type 2 diabetes in women: a prospective study. JAMA. 282(15):1433-9.

Hurley BF. (1989). Effects of resistive training on lipoprotein-lipid profiles: a comparison to aerobic exercise training. Med Sci Sports Exerc. 21(6):689-93. Review.

Ibanez J. (2005). Twice-weekly progressive resistance training decreases abdominal fat and improves insulin sensitivity in older men with type 2 diabetes. Diabetes Care. 28(3):662-7.

Ishii T. (1998). Resistance training improves insulin sensitivity in NIDDM subjects without altering maximal oxygen uptake. Diabetes Care. 21(8):1353-5.

Ishikawa-Takata K. (2003). How much exercise is required to reduce blood pressure in essential hypertensives: a dose-response study. Am J Hypertens. 16(8):629-33.

Jessup JV. (1998). The effects of endurance exercise training on ambulatory blood pressure in normotensive older adults. Geriatr Nephrol Urol. 8(2):103-9.

Joseph LJ. (1999). Differential effect of resistance training on the body composition and lipoprotein-lipid profile in older men and women. Metabolism. 1999 48(11):1474-80

Kallinen M. (1995). Aging, physical activity and sports injuries. An overview of common sports injuries in the elderly. Sports Med. Jul;20(1):41-52. Review.

Katzel LI. (1995). Effects of weight loss vs aerobic exercise training on risk factors for coronary disease in healthy, obese, middle-aged and older men. A randomized controlled trial. JAMA.274(24):1915-21.

Katzel LI. (1997). Sequential effects of aerobic exercise training and weight loss on risk factors for coronary disease in healthy, obese middle-aged and older men. Metabolism. 46(12):1441-7.
Kelley DE. (2001). Effects of exercise on glucose homeostasis in Type 2 diabetes mellitus. Med Sci Sports Exerc. 33(6 Suppl):S495-501; discussion S528-9. Review.

Kelley G. (1994). Antihypertensive effects of aerobic exercise. A brief meta-analytic review of randomized controlled trials. Am J Hypertens. 7(2):115-9.

Kelley GA. (2000). Progressive resistance exercise and resting blood pressure : A meta-analysis of randomized controlled trials. Hypertension. 35(3):838-43.

Kelley GA. (2001). Aerobic exercise and resting blood pressure in older adults: a meta-analytic review of randomized controlled trials. J Gerontol A Biol Sci Med Sci. 56(5):M298-303.

Kelley GA. (2004). Walking, lipids, and lipoproteins: a meta-analysis of randomized controlled trials. Prev Med. 38(5):651-61.

Kim JR. (2001). Effect of exercise intensity and frequency on lipid levels in men with coronary heart disease: Training Level Comparison Trial. Am J Cardiol. 87(8):942-6.

Knowler WC. (2002). Reduction in the incidence of type 2 diabetes with lifestyle intervention or metformin. N Engl J Med.;346(6):393-403.

Kokkinos PF. (1995). Miles run per week and high-density lipoprotein cholesterol levels in healthy, middle-aged men. A dose-response relationship. Arch Intern Med. 155(4):415-20.

Kokkinos PF. (1998). Effects of moderate intensity exercise on serum lipids in African-American men with severe systemic hypertension. Am J Cardiol. 81(6):732-5.

Kraus WE. (2002). Effects of the amount and intensity of exercise on plasma lipoproteins. N Engl J Med. 347(19):1483-92.

Lamarche B. (1992). Is body fat loss a determinant factor in the improvement of carbohydrate and lipid metabolism following aerobic exercise training in obese women? Metabolism. 41(11):1249-56.

Lee CD. (1999). Cardiorespiratory fitness, body composition, and all-cause and cardiovascular disease mortality in men. Am J Clin Nutr. 69(3):373-80.

Lee S. (2005). Cardiorespiratory fitness attenuates metabolic risk independent of abdominal subcutaneous and visceral fat in men. Diabetes Care 28:895-901.

LeMura LM. (2000). Lipid and lipoprotein profiles, cardiovascular fitness, body composition, and diet during and after resistance, aerobic and combination training in young women. Eur J Appl Physiol. 82(5-6):451-8.

Leon AS. (2002). Variability in the response of HDL cholesterol to exercise training in the HERITAGE Family Study. Int J Sports Med. 23(1):1-9.

Leon AS. (2000). Blood lipid response to 20 weeks of supervised exercise in a large biracial population: the HERITAGE Family Study. Metabolism. 49(4):513-20.

Leon AS. (2001). Response of blood lipids to exercise training alone or combined with dietary intervention. Med Sci Sports Exerc. 33(6 Suppl):S502-15; discussion S528-9. Review.

Lim K, Taylor L. (2005). Factors associated with physical activity among older people - a population-based study. Prev Med. Jan;40(1):33-40.

Maiorana A. (2002). Combined aerobic and resistance exercise improves glycemic control and fitness in type 2 diabetes. Diabetes Res Clin Pract. 56(2):115-23.

Manning JM. (1991). Effects of a resistive training program on lipoprotein-lipid levels in obese women. Med Sci Sports 
Exerc. 23(11):1222-6.

Matthews DR. (1985). Homeostasis model assessment: insulin resistance and beta-cell function from fasting plasma glucose and insulin concentrations in man.Diabetologia. 28(7):412-9.

Mayer-Davis EJ. (1998). Intensity and amount of physical activity in relation to insulin sensitivity: the Insulin Resistance Atherosclerosis Study. JAMA. 279(9):669-74.

Merrill JR. (1989). Hyperlipemic response of young trained and untrained men after a high fat meal. Arteriosclerosis. 9(2):217-23.

Miyatake N. (2002). Daily walking reduces visceral adipose tissue areas and improves insulin resistance in Japanese obese subjects. Diabetes Res Clin Pract. 58(2):101-7.

Miyatake N. (2003). Daily exercise lowers blood pressure and reduces visceral adipose tissue areas in overweight Japanese men. Diabetes Res Clin Pract. 62(3):149-57.

Motoyama M. (1998). Blood pressure lowering effect of low intensity aerobic training in elderly hypertensive patients. Med Sci Sports Exerc. 30(6):818-23.

Mourier A. (1997). Mobilization of visceral adipose tissue related to the improvement in insulin sensitivity in response to physical training in NIDDM. Effects of branched-chain amino acid supplements. Diabetes Care. 20(3):385-91.

Neter JE. (2003). Influence of weight reduction on blood pressure: a meta-analysis of randomized controlled trials. Hypertension. 42(5):878-84.

Ohkubo T. (2001). Effects of exercise training on home blood pressure values in older adults: a randomized controlled trial. J Hypertens. 19(6):1045-52.

Paffenbarger RS Jr. (1997). Intensity of physical activity related to incidence of hypertension and all-cause mortality: an epidemiological view. Blood Press Monit. 2(3):115-123.

Pan XR. (1997). Effects of diet and exercise in preventing NIDDM in people with impaired glucose tolerance. The Da Qing IGT and Diabetes Study. Diabetes Care. 20(4):537-44.

Pescatello LS. (2004). American College of Sports Medicine position stand. Exercise and hypertension. Med Sci Sports Exerc. 2004 Mar;36(3):533-53.

Poirier P. (2001). Exercise in weight management of obesity. Cardiol Clin. 19(3):459-70.

Pollock ML. (1994). Exercise training and prescription for the elderly. South Med J. 87(5):S88-95.

Posner JD. (1992). Low to moderate intensity endurance training in healthy older adults: physiological responses after four months. J Am Geriatr Soc. 40(1):1-7.

Pratley RE. (2000). Aerobic exercise training-induced reductions in abdominal fat and glucose-stimulated insulin responses in middle-aged and older men. J Am Geriatr Soc. 48(9):1055-61.

Reaven GM. (1988). Banting lecture 1988. Role of insulin resistance in human disease. Diabetes. 37(12):1595-607. Review.

Reynolds TH $4^{\text {th }}$. (2004). Resistance training enhances insulin-mediated glucose disposal with minimal effect on the tumor necrosis factor-alpha system in older hypertensives. Metabolism. 53(3):397-402.

Rice T. (2002). Familial aggregation of blood lipid response to exercise training in the health, risk factors, exercise training, and genetics (HERITAGE) Family Study. Circulation. 105(16):1904-8.

Ross R. (2000). Reduction in obesity and related comorbid conditions after diet-induced weight loss or exercise-induced weight loss in men. A randomized, controlled trial. Ann
Intern Med. 133(2):92-103.

Ross R. (2004). Exercise-induced reduction in obesity and insulin resistance in women: a randomized controlled trial. Obes Res. 12(5):789-98.

Ryan AS. (2000). Changes in plasma leptin and insulin action with resistive training in postmenopausal women. Int J Obes Relat Metab Disord. 24(1):27-32.

Ryan AS. (1996). Resistive training increases insulin action in postmenopausal women. Biol Sci Med Sci. 51(5):M199-205.

Sady SP. (1986). Prolonged exercise augments plasma triglyceride clearance. JAMA. 256(18):2552-5.

Saito I. (1992). Leisure time physical activity and insulin resistance in young obese students with hypertension. Am J Hypertens. 5(12 Pt 1):915-8.

Samitz G. (1991). Physical training programs and their effects on aerobic capacity and coronary risk profile in sedentary individuals. Design of a long-term exercise training program. J Sports Med Phys Fitness. 31(2):283-93.

Schwartz RS. (1995). The effects of endurance and resistance training on blood pressure. Int J Obes Relat Metab Disord. 19 Suppl 4:S52-7. Review.

Sedgwick AW. (1989). Cross-sectional and longitudinal relationships between physical fitness and risk factors for coronary heart disease in men and women: "the Adelaide 1000". J Clin Epidemiol. 42(3):189-200.

Serrano Rios M. (1998). Relationship between obesity and the increased risk of major complications in non-insulin-dependent diabetes mellitus. Eur J Clin Invest. 28 Suppl 2:14-7, discussion 17-8. Review.

Short KR. (2003). Impact of aerobic exercise training on age-related changes in insulin sensitivity and muscle oxidative capacity. Diabetes. 52(8):1888-96.

Spate-Douglas T. (1999). Exercise intensity: its effect on the high-density lipoprotein profile. Arch Phys Med Rehabil. 80(6):691-5.

Stevenson JS. (1990). Effects of moderate and low intensity long-term exercise by older adults Res Nurs Health. 13(4):209-18.

Stewart KJ. (2005). Effect of exercise on blood pressure in older persons: a randomized controlled trial. Arch Intern Med. 165(7):756-62.

Sunami Y. (1999). Effects of low-intensity aerobic training on the high-density lipoprotein cholesterol concentration in healthy elderly subjects. Metabolism. 48(8):984-8.

Szapary PO. (2003). Physical activity and its effects on lipids. Curr Cardiol Rep. 5(6):488-92. Review.

Taunton JE. (1997). Exercise for the older woman: choosing the right prescription. Br J Sports Med. 31(1):5-10.

Thompson PD. (1980). Acute effects of prolonged exercise on serum lipids. Metabolism. 29(7):662-5.

Thompson PD. (1988). Modest changes in high-density lipoprotein concentration and metabolism with prolonged exercise training. Circulation. 78(1):25-34.

Tuomilehto J. (2001). Prevention of type 2 diabetes mellitus by changes in lifestyle among subjects with impaired glucose tolerance. N Engl J Med. 344(18):1343-50.

Vessby B. (2000). Dietary fat and insulin action in humans. Br J Nutr. 83 Suppl 1:S91-6.

Weintraub MS. (1989). Physical exercise conditioning in the absence of weight loss reduces fasting and postprandial triglyceride-rich lipoprotein levels. Circulation. 79(5):1007-14.

Wilund KR. (2002). Changes in high-density lipoprotein- 
cholesterol subfractions with exercise training may be dependent on cholesteryl ester transfer protein (CETP) genotype. Metabolism. 51(6):774-8.

Wood PD. (1983). Increased exercise level and plasma lipoprotein concentrations: a one-year, randomized, controlled study in sedentary, middle-aged men. Metabolism. 32(1):31-9.

Wood PD (1988). Changes in plasma lipids and lipoproteins in overweight men during weight loss through dieting as compared with exercise. N Engl J Med. 319(18):1173-9.

Zelasko CJ. (1995). Exercise for weight loss: what are the facts? J Am Diet Assoc. 95(12):1414-7.

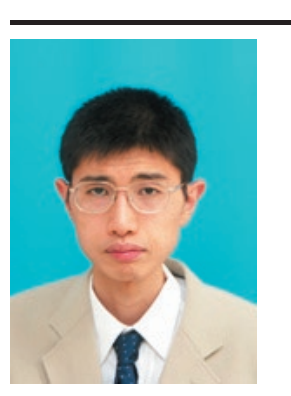

\section{Name:}

Satoru Kodama

\section{Affiliation:}

Doctoral Program in Sports Medicine, University of Tsukuba Graduate School of Comprehensive Human Sciences

Address:

1-1-1 Tennodai, Tsukuba, Ibaraki 305-8575 Japan

Brief Biographical History:

2001- Resident,Niigata City General Hospital.

2003- Doctoral Program in Sports Medicine, University of Tsukuba Graduate School of Comprehensive Human Sciences

Membership in Learned Societies:

- The Japanese Society of physical fitness

- The Japanese Society of Internal Medicine

- Japanese Society of Clinical Sports Medicine 